\title{
On the thermal gradient in the Earth's deep interior
}

\author{
M. Tirone \\ Institut für Geologie, Mineralogie und Geophysik, Ruhr-Universität Bochum, 44780 Bochum, Germany \\ Correspondence to: M. Tirone (max.tirone@gmail.com)
}

Received: 19 August 2015 - Published in Solid Earth Discuss.: 8 September 2015

Revised: 14 January 2016 - Accepted: 20 January 2016 - Published: 10 February 2016

\begin{abstract}
Temperature variations in large portions of the mantle are mainly controlled by the reversible and irreversible transformation of mechanical energy related to pressure and viscous forces into internal energy along with diffusion of heat and chemical reactions. The simplest approach to determine the temperature gradient is to assume that the dynamic process involved is adiabatic and reversible, which means that entropy remains constant in the system. However, heat conduction and viscous dissipation during dynamic processes effectively create entropy. The adiabatic and nonadiabatic temperature variation under the influence of a constant or varying gravitational field are discussed in this study from the perspective of the Joule-Thomson (JT) throttling system in relation to the transport equation for change of entropy. The JT model describes a dynamic irreversible process in which entropy in the system increases but enthalpy remains constant (at least in an equipotential gravitational field). A comparison is made between the thermal gradient from the JT model and the thermal gradient from two models, a mantle convection and a plume geodynamic model, coupled with thermodynamics including a complete description of the entropy variation. The results show that the difference is relatively small and suggests that thermal structure of the asthenospheric mantle can be well approximated by an isenthalpic model when the formulation includes the effect of the gravitational field. For non-dynamic or parameterized mantle dynamic studies, the JT formulation provides a better description of the thermal gradient than the classic isentropic formulation.
\end{abstract}

\section{Introduction}

In the Earth's deep interior, dynamic processes involving large pressure variations induce temperature changes that often are approximately described by an adiabatic gradient. The transformation of pressure-volume mechanical work into thermal heat is strictly considered adiabatic when there is no exchange of heat between the system and the surroundings, $\delta q=0$. The common simplification applied to solid Earth problems is that the process is also reversible, hence the transformation is isentropic (Lewis and Randall, 1961; Denbigh, 1971; Sandler, 1988; Turcotte and Schubert, 1982; Schubert et al., 2001). The reversible condition is in most cases an approximation; in fact, spontaneous or natural process are intrinsically irreversible, therefore $\mathrm{d} S$ is usually not zero (Lewis and Randall, 1961; Denbigh, 1971; Zemansky et al., 1975; Sandler, 1988). The irreversible entropy production in the mantle comes from various sources, mainly from heat conduction transformation of mechanical energy into internal energy by viscous forces (viscous dissipation) and chemical reactions. While determination of the entropy production from all these effects may require a full-scale dynamic thermal model (e.g Bird et al., 2002), an alternative is given by the description of the throttling process designed by the Joule-Thomson (JT) experiment (e.g. Zemansky et al., 1975) which is usually referred to as a typical example of an isenthalpic process and has been extensively discussed in the geological context by Ganguly (2008).

Waldbaum (1971) considered the adiabatic expansion in the Earth's interior, essentially assuming the formulation derived from the JT experiment. Ramberg (1971) included the effect of the gravitational potential in the treatment of irreversible decompression and Spera (1981) applied a timedependent dynamic version to metasomatic fluid flow. A detailed study of the isenthalpic formulation for irreversible 
mantle upwelling which also extended to melting processes was presented by Ganguly (2005).

However, some questions remain in particular as to whether the JT model is better suited than the isentropic formulation to describe the thermal gradient in the mantle. In addition the difference in terms of thermal or entropy change with the complete entropy description from a dynamic thermal model has not been quantified yet.

In Sect. 2 the thermodynamic and heat transport formulation is presented with focus on the entropy change and the connection to the JT formulation. In Sects. 3 and 4 a 2-D mantle convection and a 2-D thermochemical plume model combined with a chemical equilibrium approach (Gibbs free energy minimization) are used to determine the entropyrelated term and the irreversible thermal contribution. The result is then compared with the JT thermal model in order to understand whether the JT formulation could provide a reasonable description of the thermal structure of the mantle. The general outline of the numerical computation of the adiabatic thermal gradient (reversible and irreversible) in combination with a chemical equilibrium approach is described in Sect. 5, along with a series of mantle geotherms to illustrate the effect of the irreversible entropy production based on the JT formulation. Numerical details can be found in the Appendix.

\section{Thermodynamic and transport formulation of thermal change}

This section presents the formulation for the entropy change in relation to heat conduction and viscous dissipation in transport models and the relation that needs to be fulfilled for a process to be assimilated to the JT model. The general expression for the entropy change of a moving fluid in a quasi-equilibrium state is given by Bird et al. (2002):

$\rho \frac{\mathrm{D} S}{\mathrm{D} t}=-\nabla \cdot \frac{q}{T}+\sigma$

where $S$ is the entropy per unit mass, $\mathrm{D} / \mathrm{D} t$ is the substantial derivative; the first term on the right-hand side (rhs) is the rate of entropy increase by heat conduction and the second term $\sigma$ is the entropy production. The first law of thermodynamics $\mathrm{d} U=T \mathrm{~d} S-P \mathrm{~d} V$ for a small mass moving with the fluid is

$\frac{\mathrm{D} U}{\mathrm{D} t}=T \frac{\mathrm{D} S}{\mathrm{D} t}-P \frac{\mathrm{D} V}{\mathrm{D} t}$

and the transport equation for the internal energy is given by the following (Bird et al., 2002):

$\rho \frac{\mathrm{D} U}{\mathrm{D} t}=-\nabla \cdot q-P \nabla \cdot v-\tau: \nabla v$,

where the first term on the rhs is the rate of energy change by heat conduction, the second term is the reversible energy change by volume compression/expansion and the last term is the irreversible energy increase by viscous dissipation, assuming a Newtonian fluid with velocity $v$ and viscous stress tensor $\tau$. Inserting $\mathrm{D} S / \mathrm{D} t$ from Eq. (1) in (2) and combining the relation for $\mathrm{D} U / \mathrm{D} t$ with Eq. (3):

$-T \nabla \cdot \frac{q}{T}+T \sigma-\rho P \frac{\mathrm{D} V}{\mathrm{D} t}=-\nabla \cdot q-P \nabla \cdot v-\tau: \nabla v$.

Using the relation $P \nabla \cdot v=\rho P \mathrm{DV} / \mathrm{D} t$ (Sandler, 1988) and the relation

$\nabla \cdot \frac{q}{T}=\frac{1}{T} \nabla \cdot q+q \cdot \nabla \frac{1}{T}$

in Eq. (4), the entropy production $\sigma$ can be defined as

$\sigma=q \cdot \nabla \frac{1}{T}-\frac{1}{T} \tau: \nabla v$.

The effect of chemical transformations and other potential sources of entropy are ignored for simplicity or because they are negligible in the mantle (electric resistance, magnetic field, non-Newtonian fluid). The equation of change for temperature for a mass moving with the fluid can be obtained from Eq. (1) by replacing $\sigma$ with the expression given in Eq. (6) and the thermodynamic relation for $\mathrm{d} S$ as a function of $P, T, \mathrm{~d} S=C_{\mathrm{p}} / T \mathrm{~d} T-\alpha V \mathrm{~d} P$ :

$\rho C_{\mathrm{p}} \frac{\mathrm{D} T}{\mathrm{D} t}=-\nabla \cdot q-\tau: \nabla v+\alpha T \frac{\mathrm{D} P}{\mathrm{D} t}$,

where $\rho=1 / V$ has been applied. This is the standard description of temperature change that, for an inviscid fluid under adiabatic and time- and space-invariant conditions, describes an isentropic thermal gradient $\left(\mathrm{d} T /\left.\mathrm{d} P\right|_{S}=\right.$ $\left.\alpha V T / C_{\mathrm{p}}\right)$.

In the well-known Joule-Thomson experiment (e.g. Zemansky et al., 1975; Callen, 1985; Ganguly, 2008) a gas steadily flows from one subsystem to another subsystem through a porous plug. The plug avoids turbulent flow and inhomogeneous pressure distribution in both subsystems. The entire system (made of the two subsystems) is confined on both ends by two moving pistons. The pistons quasi-statically keep a predefined pressure on both sides. The whole system is thermally insulated and mass is conserved. The thermodynamic analysis shows that the process is isenthalpic, $\mathrm{d} H=0$ (Lewis and Randall, 1961; Denbigh, 1971; Zemansky et al., 1975; Callen, 1985). We can express the equation of change for the internal energy (Eq. 3) as a function of the enthalpy change by replacing $\mathrm{D} U / \mathrm{D} t$ with $\mathrm{D} H / \mathrm{D} t-\mathrm{D}(P V) / \mathrm{D} t$ :

$\rho \frac{\mathrm{D} H}{\mathrm{D} t}=-\nabla \cdot q-\tau: \nabla v+\frac{\mathrm{D} P}{\mathrm{D} t}$,

where the relation $P \nabla \cdot v=\rho \mathrm{D}(P / \rho) / \mathrm{D} t-\mathrm{D} P / \mathrm{D} t$ has been used. Therefore for an isenthalpic process $(\mathrm{D} H / \mathrm{D} t=0)$ Eq. (8) establishes the relation

$\frac{\mathrm{D} P}{\mathrm{D} t}=\nabla \cdot q+\tau: \nabla v$ 
or using the expression for the entropy production Eq. (6):

$$
\frac{\mathrm{D} P}{\mathrm{D} t}=-T \sigma+T \nabla \cdot \frac{q}{T} \text {. }
$$

Both relations show the necessary condition that must hold for an isenthalpic process. The isenthalpic condition can be verified by using Eq. (10) in the equation of change for temperature (Eq. 7) to find the equation of change for temperature $\rho C_{\mathrm{p}} \mathrm{D} T / \mathrm{D} t=(\alpha T-1) \mathrm{D} P / \mathrm{D} t$ which, in differential form, reproduces the well-known isenthalpic thermal gradient $\mathrm{d} T /\left.\mathrm{d} P\right|_{H}=(\alpha T-1) /\left(\rho C_{\mathrm{p}}\right)$ (Ramberg, 1971; Ganguly, 2008). The same relation between pressure and the entropy production (Eqs. 9 and 10) can be also obtained by expressing the thermodynamic relation given in Eq. (2) in terms of enthalpy as $\mathrm{D} H / \mathrm{D} t=T \mathrm{D} S / \mathrm{D} t+V \mathrm{D} P / \mathrm{D} t$. Assuming the isenthalpic condition, we have

$T \frac{\mathrm{D} S}{\mathrm{D} t}=-V \frac{\mathrm{D} P}{\mathrm{D} t}$,

then Eq. (10) can be found by using Eq. (1) to replace $\mathrm{D} S / \mathrm{D} t$ in the above expression.

In a varying gravitational field, the isenthalpic condition should be replaced by $\mathrm{d} H=V \mathrm{~d} P_{\mathrm{g}}$ (Dodson, 1971; Ramberg, 1971), where $\mathrm{d} P_{\mathrm{g}}=\rho g \mathrm{~d} z$. Then the expression for entropy equivalent to Eq. (11) is

$T \frac{\mathrm{D} S}{\mathrm{D} t}=V\left(\frac{\mathrm{D} P_{\mathrm{g}}}{\mathrm{D} t}-\frac{\mathrm{D} P}{\mathrm{D} t}\right)$

and the equivalent to Eq. (9) relating the pressure changes to entropy changes is

$\frac{\mathrm{D} P}{\mathrm{D} t}-\frac{\mathrm{D} P_{\mathrm{g}}}{\mathrm{D} t}=\nabla \cdot q+\tau: \nabla v$.

The dynamic thermal model (Eq. 7) is not affected by these two equations, however if the conditions are such that in Eq. 13 the difference of the pressure changes on the lefthand side (lhs) and the entropy-related terms on the rhs are close to be equal, then the thermal process can be assimilated to a JT model in a varying gravitation field. If this is the case, Eq. (12) can be used to find the thermal gradient for the JT model including the gravitational effect. Under space- and time-invariant conditions, Eq. (12) reduces to $T \mathrm{~d} S=V \mathrm{~d} P_{\mathrm{g}}-V \mathrm{~d} P$, and by expressing the entropy change $\mathrm{d} S$ as $\mathrm{d} S=C_{\mathrm{p}} / T \mathrm{~d} T-\alpha V \mathrm{~d} P$, the thermal gradient can be described by the following equation:

$$
\left.\frac{\mathrm{d} T}{\mathrm{~d} P}\right|_{H_{\mathrm{g}}}=\frac{(\alpha T-1) V}{C_{\mathrm{p}}}+\frac{1}{C_{\mathrm{p}}} \frac{\mathrm{d} P_{\mathrm{g}}}{\mathrm{d} P} .
$$

The interesting aspect of the JT thermal model is that a pressure gradient (difference), which fundamentally defines dynamic flow, is naturally included. In addition entropy increases only when the pressure is not equal to the pressure generated by the gravitational field $P_{\mathrm{g}}$. The pressure change

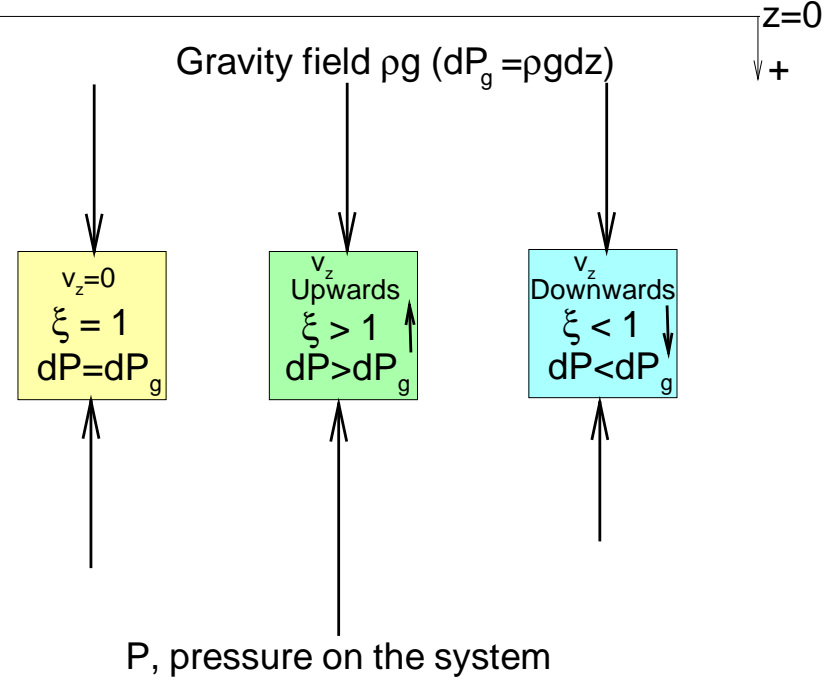

Figure 1. Vertical pressure components acting on the system. Vertical motion is the result of the difference between the gravitational pressure force $P_{\mathrm{g}}$ and the vertical pressure force $P$ acting on the system.

can be related to the gravitational pressure by a scaling factor $\zeta$ so that $\mathrm{d} P=\zeta \rho g \mathrm{~d} z=\zeta \mathrm{d} P_{\mathrm{g}}$. Using this scaling factor in Eq. (14), the following expression for temperature changes with depth under adiabatic irreversible conditions in a varying gravitational field is

$\left.\frac{\mathrm{d} T}{\mathrm{~d} z}\right|_{H_{\mathrm{g}}}=\frac{g}{C_{\mathrm{p}}}(1-\zeta)+\zeta\left(\frac{g T \alpha}{C_{\mathrm{p}}}\right)$.

This equation is the same as the corresponding expression derived by Ganguly (2005, Eq. 16) after setting $\zeta=\rho_{\mathrm{r}} / \rho$, where $\rho_{\mathrm{r}}$ is the density of the ambient mantle at rest (so that $\mathrm{d} P=\rho_{\mathrm{r}} g \mathrm{~d} z$ ), and the acceleration term is ignored. Using the scaling factor $\zeta$, the irreversible entropy production assumes the following form:

$\mathrm{d} S=-V \mathrm{~d} P_{\mathrm{g}}(\zeta-1) / T$.

The condition of hydrostatic equilibrium is obtained when $\zeta=1$ and no entropy is produced. If $\zeta>1$ (pressure gradient is greater than the gradient of the gravitational pressure), the system moves upwards (Fig. 1). Noticeably the increase of entropy moving upwards $(\mathrm{d} S / \mathrm{d} z<0)$ is consistent with a spontaneous process. Conversely, when $\zeta<1$, entropy increases at greater depths $(\mathrm{d} S / \mathrm{d} z>0)$ and the material spontaneously moves downwards.

\section{Mantle geotherms in a convective mantle}

A convection model is used in this section to understand whether the condition given by Eq. (12) or (13) is fulfilled 
to the extent that would justify the use of the JT model to describe the thermal gradient in the mantle. The results summarized in Fig. 2 are based on a 2-D compressible flow model heated from below and coupled to a thermodynamic formulation associated with Saxena's database (Saxena, 1996) in the system $\mathrm{MgO}-\mathrm{FeO}-\mathrm{SiO}_{2}$ to computed density and heat capacity. The oxides' abundance, defined as $\mathrm{MgO}=44.72$, $\mathrm{FeO}=9.48, \mathrm{SiO} 2=45.8(\mathrm{wt} \%)$, approximately describes a peridotite bulk composition. The dynamic transport equations for a compressible flow can be found in Schubert et al. (2001). The details of the numerical solution are given in Tirone et al. (2009). Viscosity is set to $\left(1 \times 10^{22} \mathrm{Pas}\right)$, thermal conductivity is $3 \mathrm{~W} \mathrm{~m}^{-1} \mathrm{~K}^{-1}$ and the bottom temperature is $3327^{\circ} \mathrm{C}$. The temperature at the base of the model is set in a way that the model does not create an unrealistic exceedingly hot upper mantle. The dynamic thermal model does not include any viscous dissipation or adiabatic reversible terms in the transport equation which is simply given by $\rho C_{\mathrm{p}} \mathrm{D} T / \mathrm{D} t=-\nabla \cdot q$. The additional terms in the thermal equation are computed in a second stage using the parameters from the dynamic model. This simplified approach implies that there is no coupling between the parameters used to compute the thermal gradients and the computed thermal gradients. On the right middle panel of Fig. 2, the terms $\left(v \cdot\left(\nabla P-\nabla P_{\mathrm{g}}\right)\right)$ and $(\nabla \cdot q+\tau: \nabla v)$ are used to assess whether the irreversible contribution to the thermal gradient from the JT model is comparable to the irreversible effect from heat conduction and viscous dissipation (see Eq. 13) along the upwelling and downwelling flow directions. Assuming a steady state, the vertical component of the thermal effect $\Delta T_{z}$ added to the isentropic gradient is given by the integration of $\left(v \cdot\left(-\nabla P+\nabla P_{\mathrm{g}}\right)\right) \Delta z /\left(\rho C_{\mathrm{p}} v_{z}\right)$ and $(-\nabla \cdot q-\tau: \nabla v) \Delta z /\left(\rho C_{\mathrm{p}} v_{z}\right)$ for the JT model and general entropy case respectively. The result for the downwelling and upwelling flow are shown in the lower panel of Fig. 2. For this particular mantle convection model it appears that the difference between the two thermal gradients is quite small. It is also quite evident that the JT model is a better representation of the thermal variations than the one offered by the isentropic formulation. The ratio between the flow pressure and the gravitational pressure $P_{\mathrm{g}}$ along two vertical sections extracted from the model (middle left panel of Fig. 2) is the necessary information that can be used to compute the JT thermal gradient in non-dynamic models (see Sect. 5).

\section{Thermal gradient of a mantle plume}

In this section, a detailed geodynamic model of a thermal plume is used for a further comparison with the thermal gradient given by the JT model. The general description of the coupling between the geodynamic model and the thermodynamic formulation has been discussed elsewhere (Tirone et al., 2009). The transport equation for temperature change in this model includes an additional heat source term to de-
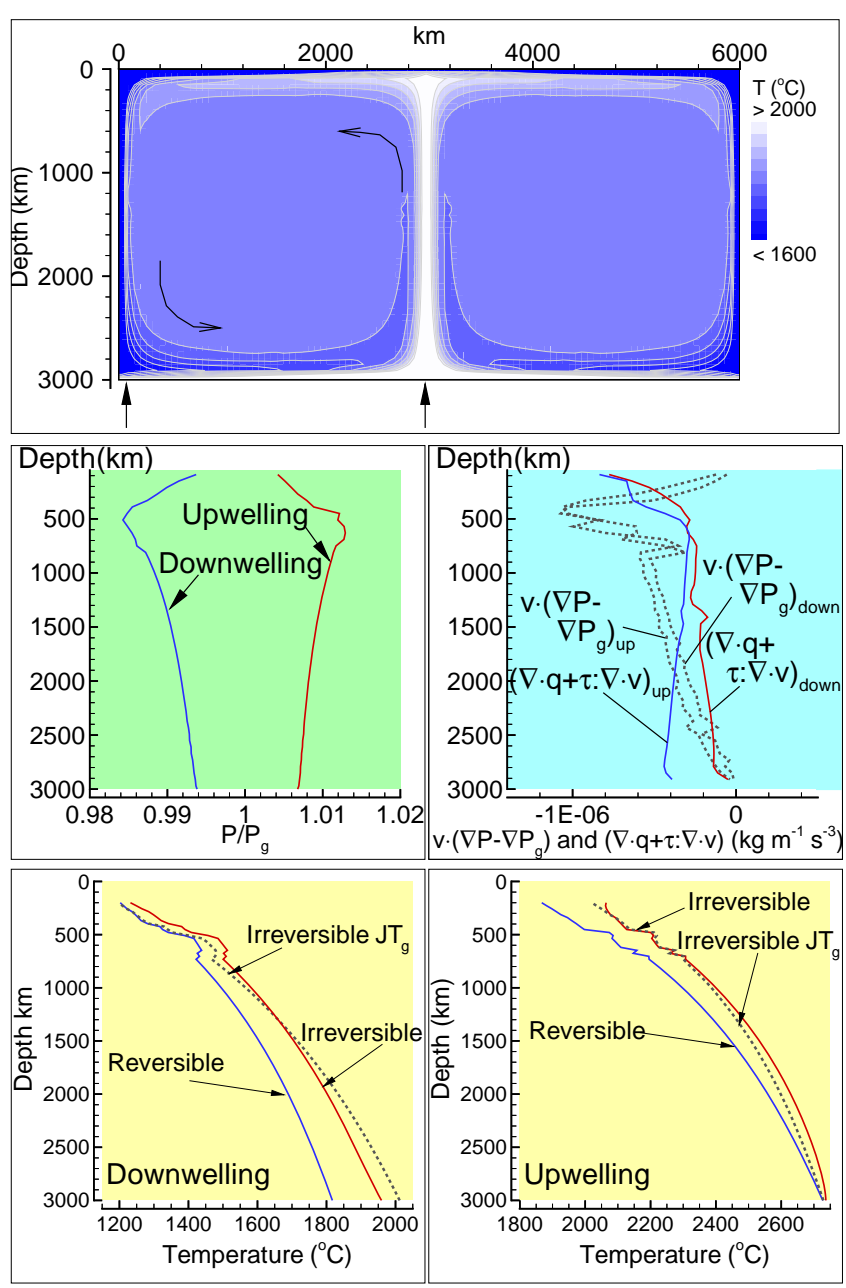

Figure 2. The upper panel shows temperature for an isoviscous mantle convection simulation (heated from below) coupled with a thermodynamic model. Temperature at the bottom is set to $3327^{\circ} \mathrm{C}$. In the geodynamic model the thermal field does not include the reversible adiabatic and viscous dissipation effects. The middle left panel shows the ratio between the flow pressure and the gravitational pressure $P_{\mathrm{g}}=\rho g \mathrm{~d} z$. The middle right panel shows the irreversible contribution to the thermal gradient given by heat conduction and viscous dissipation (solid lines) and the irreversible contribution in the JT formulation including the gravitational effect (dashed lines; see Eq. 13). The lower panel shows the thermal gradient computed from the isentropic formulation (reversible), the irreversible formulation by adding heat conduction and viscous dissipation (irreversible) and the JT formulation with gravity (dashed lines, irreversible $\mathrm{JT}_{\mathrm{g}}$ ).

scribe the contribution of chemical transformations:

$\rho C_{\mathrm{p}} \frac{\mathrm{D} T}{\mathrm{D} t}=-\nabla \cdot q-\tau: \nabla v+\alpha T \frac{\mathrm{D} P}{\mathrm{D} t}+T \rho \sum S_{i} \frac{\mathrm{D} n_{i}}{\mathrm{D} t}$,

where the moles of the mineral components at equilibrium $n_{i}$ and the molar entropy $S_{i}$ are retrieved from the thermodynamic computation. For practical purposes the substantial derivative $\mathrm{D} n_{i} / \mathrm{D} t$ has been solved considering only the ad- 
vective vertical component. The temperature at the bottom of the 2-D numerical model is set to $2827^{\circ} \mathrm{C}$. This temperature has been chosen a posteriori based on the thermal structure of the plume in the upper mantle that should not be too far off from the thermal conditions for melting a dry peridotite. The viscosity is defined by a model which depends on pressure, temperature and mineralogical assemblage. Thermal conductivity in the upper mantle is set to $4 \mathrm{~W} \mathrm{~m}^{-1} \mathrm{~K}^{-1}$. In the lower mantle the thermal conductivity is defined by the model of Manthilake et al. (2011), and assumes the following form: $k=4.7(700 / T)^{0.21}(\rho / 4400)^{4}$. The numerical grid spacing is $\Delta x=10 \mathrm{~km}, \Delta z=10$. All other parameters that are needed in the dynamic model are defined by the thermodynamic formulation using Saxena's database (Saxena, 1996) supplemented with the thermodynamic parameters for post-perovskite (ppv), retrieved by summarizing several experimental data and previous studies (Murakami et al., 2004; Tsuchiya et al., 2005; Hirose, 2006; Shieh et al., 2006; Spera et al., 2006; Tateno et al., 2007; Komabayashi et al., 2008; Shim, 2008; Andrault et al., 2010; Dorfman et al., 2013).

The thermodynamic parameters for $\mathrm{MgSiO}_{3}$-ppv and $\mathrm{FeSiO}_{3}$-ppv are as follows:

- $\left(\mathrm{MgSiO}_{3}\right.$-ppv $)$ reference enthalpy, entropy and molar volume at $300 \mathrm{~K}, 1$ bar: $\Delta \mathrm{H}_{\mathrm{f}}\left(T_{\mathrm{r}}, P_{\mathrm{r}}\right)=-1405894$ $\left(\mathrm{j} \mathrm{mol}^{-1}\right), \quad S\left(T_{\mathrm{r}}, P_{\mathrm{r}}\right)=77.60 \quad\left(\mathrm{j} \mathrm{mol}^{-1} \mathrm{~K}^{-1}\right)$, $v_{0}\left(T_{\mathrm{r}}, P_{\mathrm{r}}\right)=2.4447 \quad\left(\mathrm{j} \mathrm{mol}^{-1} \mathrm{bar}^{-1}\right)$; heat capacity coefficients: $k_{1}=139.7, \quad k_{2}=0.8300 \mathrm{e}-5$, $k_{3}=-4410000, \quad k_{4}=0, \quad k_{5}=0.1038 \mathrm{e} 9, \quad k_{6}=0$, $k_{7}=-10346 ; \quad$ thermal expansion coefficients: $a_{1}=4.4084 \mathrm{e}-5, \quad a_{2}=-8.8066 \mathrm{e}-10, \quad a_{3}=-0.8967 \mathrm{e}-$ 2 , $\quad a_{4}=1.4107$; bulk modulus coefficients: $v_{1}=232.3 e 4, \quad a_{2}=-0.2885 \mathrm{e} 3, \quad v_{3}=1.4162 \mathrm{e}-2$ and $\mathrm{d} K / \mathrm{d} P(T=300 \mathrm{~K})=3.84, d^{2} K / \mathrm{d} P \mathrm{~d} T=1 \mathrm{e}-5$;

- $\left(\mathrm{FeSiO}_{3}\right.$-ppv) reference enthalpy, entropy and molar volume at $300 \mathrm{~K}, 1$ bar: $\Delta H_{\mathrm{f}}\left(T_{\mathrm{r}}, P_{\mathrm{r}}\right)=-1059880$ $\left(\mathrm{j} \mathrm{mol}^{-1}\right), \quad S\left(T_{\mathrm{r}}, P_{\mathrm{r}}\right)=64.07 \quad\left(\mathrm{j} \mathrm{mol}^{-1} \mathrm{~K}^{-1}\right)$, $v_{0}\left(T_{\mathrm{r}}, P_{\mathrm{r}}\right)=2.7382 \quad\left(\mathrm{j} \mathrm{mol}^{-1} \mathrm{bar}^{-1}\right)$; heat capacity coefficients: $k_{1}=139.7, \quad k_{2}=0.8300 \mathrm{e}-5$, $k_{3}=-4410000, \quad k_{4}=0, \quad k_{5}=0.1038 e 9, \quad k_{6}=0$, $k_{7}=-10346$; thermal expansion coefficients: $a_{1}=4.4084 \mathrm{e}-5, \quad a_{2}=-8.8066 \mathrm{e}-10, \quad a_{3}=-0.8967 \mathrm{e}-$ $2, \quad a_{4}=1.4107$; bulk modulus coefficients: $v_{1}=153.7 e 4, \quad a_{2}=-0.2885 \mathrm{D} 3, \quad v_{3}=1.4162 \mathrm{e}-2$ and $\mathrm{d} K / \mathrm{d} P(T=300 \mathrm{~K})=4.24, d^{2} K / \mathrm{d} P \mathrm{~d} T=5.2 \mathrm{e}-5$.

The following equations apply for heat capacity ( 1 bar): $C_{p}=k_{1}+k_{2} T+k_{3} / T^{2}+k_{4} T^{2}+k_{5} / T^{3}+k_{6} / T^{1 / 2}+k 7 / T$ $\left(\mathrm{j} \mathrm{mol}^{-1} \mathrm{~K}^{-1}\right.$ ); thermal expansion (1 bar) $\alpha=a_{1}+a_{2} T+$ $a_{3} / T+a_{4} / T^{2}\left(\mathrm{~K}^{-1}\right)$; bulk modulus (1 bar) $K=v_{1}+$ $v_{2} T+v_{3} T^{2}$ (bar); derivative of the bulk modulus with pressure $(1$ bar $) \mathrm{d} K / \mathrm{d} P=\mathrm{d} K / \mathrm{d} P(300 \mathrm{~K})+\mathrm{d}^{2} K / \mathrm{d} P \mathrm{~d} T(T-$ $300) \ln (T / 300)$. Mixing between Mg-ppv and Fe-ppv is assumed to be ideal.

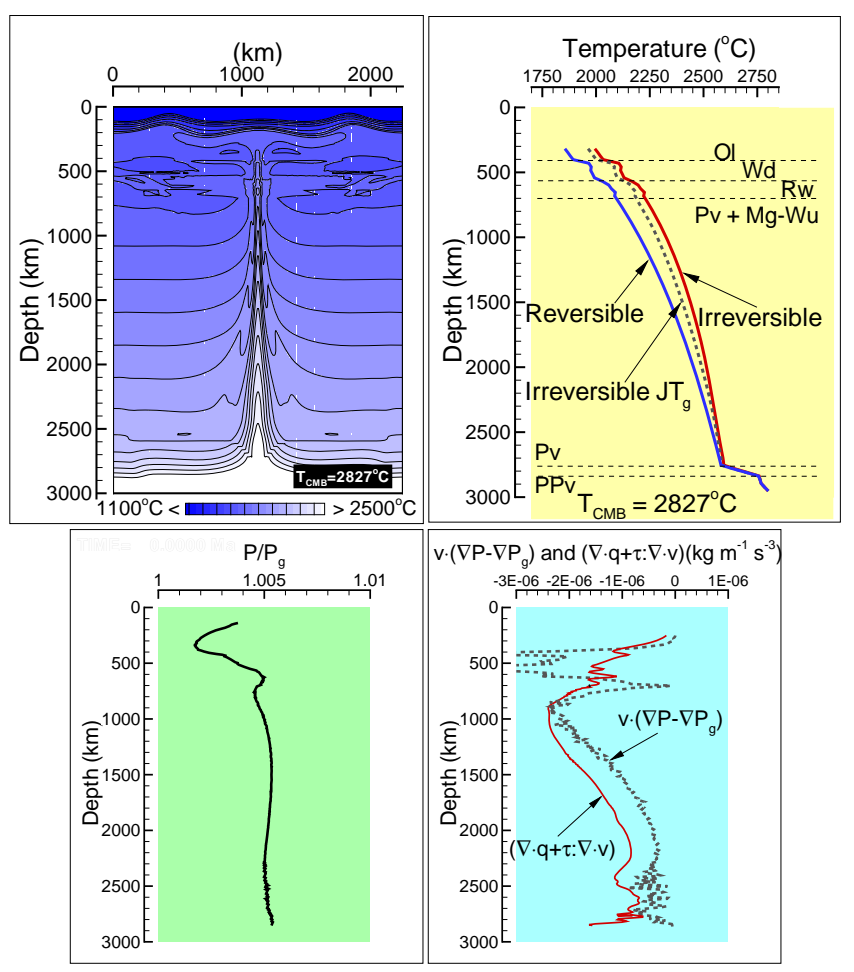

Figure 3. The top left panel shows the dynamic model of a thermal plume coupled with a thermodynamic formulation, including the irreversible effect of heat conduction, viscous dissipation and chemical transformations. The right panel shows the comparison of the thermal profile along the plume vertical section between the numerical model that includes the irreversible effects (from the simulation shown on the left panel) and the thermal gradient including the JT irreversible effect (dashed line). The reversible thermal profile is obtained by subtracting the integral of $(-\nabla \times q-\tau: \nabla v) \Delta z /\left(\rho C_{\mathrm{p}} v_{z}\right)$ from the dynamic thermal model. The lower left panel shows the ratio between the flow pressure and the gravitational pressure. The lower right panel shows the irreversible contribution to the thermal gradient given by heat conduction and viscous dissipation for the plume model (solid line) and irreversible contribution in the JT formulation, including the gravitational effect (dashed line).

The bulk composition is the same that was defined for a peridotite in Sect. 3. Figure 3 (upper left panel) shows the thermal structure of the plume. The upper right panel includes the vertical thermal profile approximately at the centre of the plume, including the relevant phase transition boundaries. In addition the dashed line illustrates the thermal profile obtained using the JT formulation with the gravitational effect. This result is obtained by subtracting the integral term related to the irreversible entropy effect from the dynamic thermal model $(-\nabla \cdot q-\tau: \nabla v) \Delta z /\left(\rho C_{\mathrm{p}} v_{z}\right)$ and by adding the integral term related to the JT irreversible effect $\left(v \cdot\left(-\nabla P+\nabla P_{\mathrm{g}}\right)\right) \Delta z /\left(\rho C_{\mathrm{p}} v_{z}\right)$, where only the vertical component of the thermal change has been considered. The reversible thermal gradient is obtained by applying just the subtraction operation. The result is that the two irreversible 

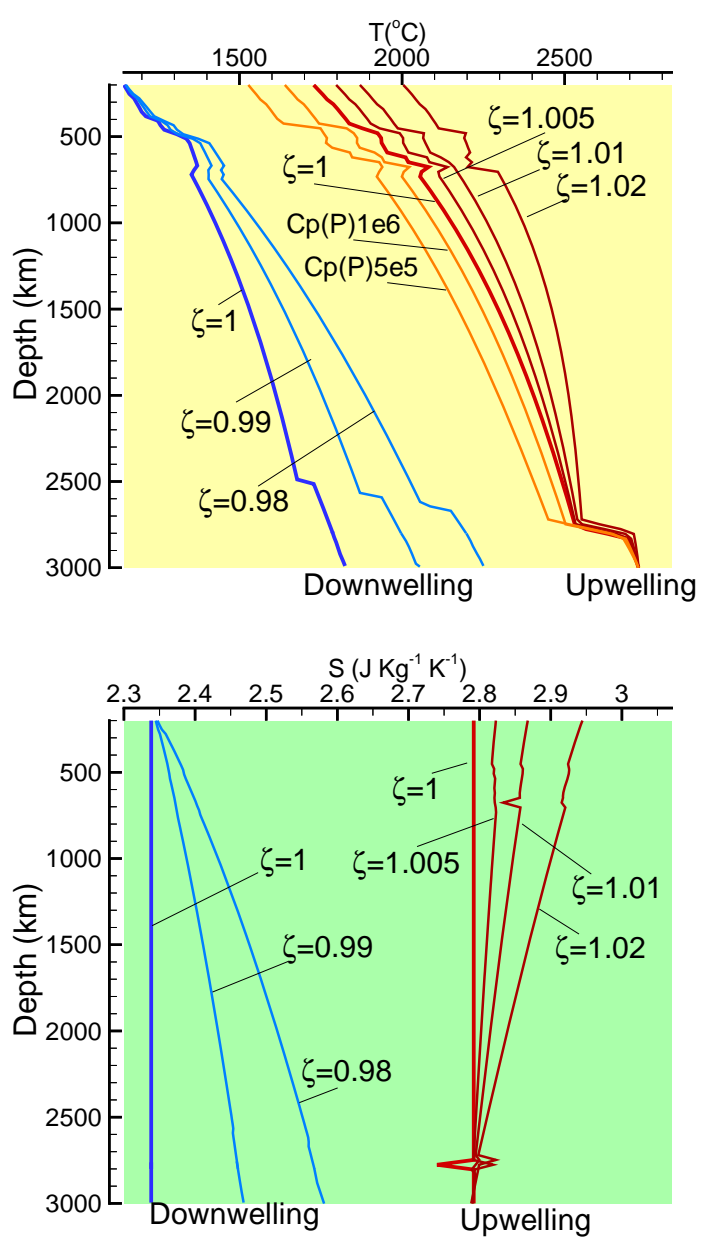

Figure 4. The upper panel shows the reversible and JT irreversible adiabatic temperature gradients in the Earth's mantle for upwelling ( $T$ at the bottom is $2727^{\circ} \mathrm{C}$ ) and downwelling ( $T$ at the top is $1150^{\circ} \mathrm{C}$ ). The lower panel shows the change of entropy with depth. All cases assume the heat capacity at 1 bar except the profiles with the label $C_{\mathrm{p}}(P)$ (see main text for further details). Bold lines highlight the isentropic case.

thermal profiles are very similar; in fact, the change of the entropy-related terms (lower right panel, Fig. 3) is quite similar. Oscillations in particular at the pv-ppv boundary and around the transition zone are the consequence of the effect of the chemical transformations on the numerical computation of the heat flux and pressure gradients. The pressure ratio $P / P_{\mathrm{g}}$ (lower left panel) can be used to compute the thermal gradient based on the JT model without performing a fullscale dynamic simulation, as shown in the next section.

\section{Mantle geotherms}

The computation of the adiabatic thermal gradient in the Earth's deep interior involves the determination at discrete depth intervals of (1) temperature, (2) pressure and (3) equi- librium mineralogical assemblage. The numerical details relative to the computation of the temperature from the relevant thermodynamic quantities are discussed in the Appendix. The thermodynamic properties and bulk composition have been defined in the previous sections. The whole algorithm can be briefly summarized as follows. With an initial guess of the density at each grid point, the gravitational pressure is computed starting at the uppermost point where the pressure and depth are predefined. Then, after computing the entropy, enthalpy and the additional functions $S^{*}$ and $H^{*}$ at the deepest point (Eqs. A1-A4, and A5, in Appendix A), the computation of the functions $S^{*}$ and $H^{*}$ (Eq. A12 for $S^{*}$ and similar for $\left.H^{*}\right)$ is combined with a Gibbs free energy minimization to determine the equilibrium assemblage and the temperature at each grid point simultaneously. Once the initial temperature profile versus depth has been determined, the gravitational pressure is re-evaluated with the updated densities along with pressure (using the scaling factor $\zeta$ ) and the procedure continues until no significant variations of the pressure at any depth are observed between two iterations.

The algorithm just outlined is applied to compute the adiabatic gradient in the mantle convective region. The thermodynamic database for the system $\mathrm{MgO}-\mathrm{FeO}-\mathrm{SiO}_{2}$ (Saxena, 1996 ) with the addition of data for post-perovskite (see previous section) is used in the Gibbs free energy minimization and additional thermodynamic calculations. The depth at the bottom is $3000 \mathrm{~km}$; the temperature at this depth is set to $2727^{\circ} \mathrm{C}$ for the upwelling. This starting temperature is within a reasonable range, consistent with the expected intersection of the plume temperature with the solidus of a peridotite in the upper mantle. For the downwelling thermal gradient the depth at the top is $200 \mathrm{~km}$; at this point the pressure is set to $62 \mathrm{kbar}$ and the temperature is fixed at $1150^{\circ} \mathrm{C}$. It should represent the temperature at some intermediate depth in a hypothetical subducting slab. This is all the information needed to compute the isentropic adiabatic gradient. To evaluate the irreversible adiabatic effect, the scaling factor $\zeta$ relating $P$ to $P_{\mathrm{g}}$ has to be specified, e.g. lower than 1.0 in case of mantle upwelling and greater than 1.0 in case of downward flow. For simplicity it is assumed to be constant at any depth, although it can vary, as has been shown in the previous sections. Figure 4 summarizes the results. On the upper panel the isentropic geotherm is plotted, along with a series of irreversible geotherms for upwelling which show that the adiabatic thermal gradient gets steeper as the scaling factor increases. These geotherms are computed, taking the heat capacity value at the given temperature and 1 bar. As discussed in Appendix A this is not exactly correct. Two additional isentropic geotherms are computed assuming a linear pressure dependence of the heat capacity from 1 bar to a predefined limit pressure (set to 500 and $1000 \mathrm{kbar}$ ) at which $C_{\mathrm{p}}$ reaches the Dulong-Petit limit. For example when the pressure limit is set to $1000 \mathrm{kbar}$, the heat capacity would vary with pressure according to the following relation: $C_{\mathrm{p}}(\mathrm{DP})-$ $\left[C_{\mathrm{p}}(\mathrm{DP})-C_{\mathrm{p}}(1 \mathrm{bar})\right] \times(1000 \mathrm{kbar}-P) / 1000$, where $C_{\mathrm{p}}(\mathrm{DP})$ 
is the value of the heat capacity at the Dulong-Petit limit. The linear assumption should at least give a qualitative sense of the pressure effect on the heat capacity and the geotherm. The result in Fig. 4 for the isentropic case $(\zeta=1)$ suggests a decrease of the thermal gradient. The lower panel of Fig. 4 illustrates the entropy production for the irreversible cases. Geotherms are also computed for downwelling, assuming an isentropic gradient and two irreversible gradients with $\zeta$ equal to 0.99 and 0.98 . The effect of the irreversible entropy production in this case is to decrease the thermal gradient.

The JT model seems to offer a better estimate of the thermal structure than the isentropic model because it accounts, to a large extent, for the entropy increase that would be observed from a full-scale dynamic thermal model.

\section{Conclusions}

The main objective of this study is to better understand whether the irreversible entropy production in the dynamic mantle can be assimilated to the entropy related to the JT model. This appears to be the case based on the two geodynamic models used in this study; in fact the thermal structure of the mantle could be assumed to follow, to a large extent, an isenthalpic model when the gravitational effect is included. The thermal gradient is closely related to the thermal gradient that would be obtained by a full-scale dynamic thermal model, therefore the model represents a better alternative to the isentropic formulation when applied to non-dynamic or parameterized thermal models. The formulation based on the JT model is relatively simple and, in comparison to the isentropic formulation, requires only one additional term (scaling factor $\zeta$ or pressure ratio).
The concept of mantle potential temperature introduced by McKenzie and Bickle (1988) is defined by the projection of an isentropic mantle adiabat to the surface. The utility of this concept lies in the notion that different parcels of rocks displaced vertically from different depths on an isentropic adiabat would intersect the solidus at the same depth. Ganguly $(2005,2008)$ discussed the limitation of this concept since different parcels of upwelling material from the same isentropic adiabat could intersect the solidus at different temperatures because of irreversible thermodynamic effects. This is further emphasized in the present study (Figs. 2, 3, 4). Figures 2 and 3 show that the potential temperature could be misleading in terms of conveying the extent of partial melting of an upwelling mantle from the core-mantle boundary, while Fig. 4 illustrates the $P-T$ trajectories of rocks upwelling from the core-mantle boundary and intersecting the Earth's surface at different temperatures, depending on the scaling factor $\zeta$. 


\section{Appendix A: Numerical procedure to compute the adiabatic thermal gradient}

The chemical equilibrium computation is based on a Gibbs free energy minimization in which pressure, temperature and bulk composition are given as input quantities. Gravitational pressure is determined by the numerical integration of $\rho g \mathrm{~d} z$, where the density is known from the equilibrium mineralogical assemblage. The computational procedure starts by evaluating the molar entropy and the enthalpy of the system at the deepest point where temperature is assumed to be known:

$$
\begin{aligned}
S_{\mathrm{m}}\left(P_{\mathrm{b}}, T_{\mathrm{b}}\right)= & S_{\mathrm{m}}(1 \mathrm{bar}, 298 \mathrm{~K})+\left.\int_{298 \mathrm{~K}}^{T_{\mathrm{b}}} \frac{C_{P_{\mathrm{m}}}}{T} \mathrm{~d} T\right|_{1 \mathrm{bar}} \\
& -\left.\left.\left.\int_{1 \mathrm{bar}}^{P_{\mathrm{b}}} \alpha_{\mathrm{m}} V_{\mathrm{m}} \mathrm{d} P\right|_{T_{\mathrm{b}}}\right|_{\mathrm{b}} C_{P_{\mathrm{m}}} \mathrm{d} T\right|_{1 \mathrm{bar}}+ \\
H_{\mathrm{m}}\left(P_{\mathrm{b}}, T_{\mathrm{b}}\right)= & H_{\mathrm{m}}(1 \mathrm{bar}, 298 \mathrm{~K})+\left.\int_{P_{\mathrm{b}}}^{P_{\mathrm{b}}} C_{P_{\mathrm{b}}} V_{T_{\mathrm{b}}} \mathrm{d} P\right|_{T_{\mathrm{b}}}-\left.T_{\mathrm{b}} \int_{\mathrm{m}} V_{\mathrm{m}} \mathrm{d} P\right|_{\text {bar }},
\end{aligned}
$$

where the subscript $b$ stands for the bottom point and the subscript $\mathrm{m}$ for the molar properties of a particular mineral component in the equilibrium assemblage. Two additional quantities $S^{*}$ or $H^{*}$, derived from the relations $\mathrm{d} S^{*}=$ $C_{\mathrm{p}} / T \mathrm{~d} T-\alpha V \mathrm{~d} P+V / T \mathrm{~d} P-V \rho g / T \mathrm{~d} z$ and $\mathrm{d} H^{*}=C_{\mathrm{p}} \mathrm{d} T+$ $(1-\alpha T) V \mathrm{~d} P-V \rho g \mathrm{~d} z$, are also computed at the bottom point:

$$
\begin{aligned}
& S_{\mathrm{m}}^{*}\left(P_{\mathrm{b}}, T_{\mathrm{b}}\right)=S_{\mathrm{m}}^{*}(1 \mathrm{bar}, 298 \mathrm{~K})+\left.\int_{298 \mathrm{~K}}^{T_{\mathrm{b}}} \frac{C_{P_{\mathrm{m}}}}{T} \mathrm{~d} T\right|_{1 \text { bar }}(\mathrm{A} 3) \\
& -\left.\int_{1 \text { bar }}^{P_{\mathrm{b}}} \alpha_{\mathrm{m}} V_{\mathrm{m}} \mathrm{d} P\right|_{T_{\mathrm{b}}}+\left.\frac{1}{T_{\mathrm{b}}} \int_{1 \text { bar }}^{P_{\mathrm{b}}} V_{\mathrm{m}} \mathrm{d} P\right|_{T_{\mathrm{b}}}-\left.\frac{1}{T_{\mathrm{b}}} \int_{1 \text { bar }}^{P_{\mathrm{b}}} V_{\mathrm{m}} \mathrm{d} P_{\mathrm{g}}\right|_{T_{\mathrm{b}}}
\end{aligned}
$$

and

$$
\begin{aligned}
& H_{\mathrm{m}}^{*}\left(P_{\mathrm{b}}, T_{\mathrm{b}}\right)=H_{\mathrm{m}}^{*}(1 \mathrm{bar}, 298 \mathrm{~K})+\left.\int_{298 \mathrm{~K}}^{T_{\mathrm{b}}} C_{P_{\mathrm{m}}} \mathrm{d} T\right|_{1 \mathrm{bar}} \\
& +\left.\int_{1 \text { bar }}^{P_{\mathrm{b}}} V_{\mathrm{m}} \mathrm{d} P\right|_{T_{\mathrm{b}}}-\left.T_{\mathrm{b}} \int_{1 \text { bar }}^{P_{\mathrm{b}}} \alpha_{\mathrm{m}} V_{\mathrm{m}} \mathrm{d} P\right|_{T_{\mathrm{b}}}-\left.\int_{1 \text { bar }}^{P_{g_{\mathrm{b}}}} V_{\mathrm{m}} \mathrm{d} P_{\mathrm{g}}\right|_{T_{\mathrm{b}}},
\end{aligned}
$$

where $\mathrm{d} P_{\mathrm{g}}$, is defined as $\rho g \mathrm{~d} z$. The relation between $\mathrm{d} P$ and $\mathrm{d} P_{\mathrm{g}}$, introduced in Sect. 2 , is $\mathrm{d} P=\zeta \mathrm{d} P_{\mathrm{g}}$ and $\zeta$ is the predefined scaling factor. The total thermodynamic properties are evaluated using

$\phi(P, T)=\sum_{\mathrm{m}} n_{\mathrm{m}} \phi_{\mathrm{m}}(P, T)$,

where $\phi$ is $S, H, S^{*}$ or $H^{*}$, and $n$ is the number of moles of the component at equilibrium obtained from the Gibbs free energy minimization. The functions $S^{*}$ and $H^{*}$ are the reference quantities that need to be maintained constant at every depth point. To evaluate these quantities at any depth, the starting point is the differential expression of the total properties:

$$
\begin{aligned}
\mathrm{d} S= & \sum_{\mathrm{m}} n_{\mathrm{m}}\left(\frac{C_{P_{\mathrm{m}}}}{T} \mathrm{~d} T-\alpha_{\mathrm{m}} V_{\mathrm{m}} \mathrm{d} P\right)+\sum_{\mathrm{m}} S_{\mathrm{m}} \mathrm{d} n_{\mathrm{m}} \\
\mathrm{d} H= & \sum_{\mathrm{m}} n_{\mathrm{m}}\left(C_{P_{\mathrm{m}}} \mathrm{d} T+\left(1-\alpha_{\mathrm{m}} T\right) V_{\mathrm{m}} \mathrm{d} P\right) \\
& +\sum_{\mathrm{m}} H_{\mathrm{m}} \mathrm{d} n_{\mathrm{m}} \\
\mathrm{d} S^{*}= & \sum_{\mathrm{m}} n_{\mathrm{m}}\left(\frac{C_{P_{\mathrm{m}}}}{T} \mathrm{~d} T-\alpha_{\mathrm{m}} V_{\mathrm{m}} \mathrm{d} P+\frac{V_{\mathrm{m}}}{T} \mathrm{~d} P-\frac{V_{\mathrm{m}}}{T} \mathrm{~d} P_{\mathrm{g}}\right) \\
& +\sum_{\mathrm{m}} S_{\mathrm{m}} \mathrm{d} n_{\mathrm{m}} \\
\mathrm{d} H^{*}= & \sum_{\mathrm{m}} n_{\mathrm{m}}\left(C_{P_{\mathrm{m}}} \mathrm{d} T+\left(1-\alpha_{\mathrm{m}} T\right) V_{\mathrm{m}} \mathrm{d} P-V_{\mathrm{m}} \mathrm{d} P_{\mathrm{g}}\right) \\
& +\sum_{\mathrm{m}} H_{\mathrm{m}} \mathrm{d} n_{\mathrm{m}} .
\end{aligned}
$$

The equations are integrated over two depth intervals, for example from $z+1$ to $z$. Integration of $S^{*}$ (Eq. A8) gives

$$
\begin{aligned}
S^{*}\left(P_{z}, T_{z}\right)= & \sum_{\mathrm{m}} n_{\mathrm{m}_{z}} S_{\mathrm{m}_{z}}^{*}=\sum_{\mathrm{m}} n_{\mathrm{m}_{z+1}} S_{\mathrm{m}_{z+1}}^{*}+\sum_{\mathrm{m}} n_{\mathrm{m}_{z}} \\
& \left\{\left.\frac{C_{P_{\mathrm{m}_{z}}}}{T_{z}}\left(T_{z}-T_{z+1}\right)\right|_{\left(P_{z+1}\right)}\right. \\
& -\left[\int_{1 \mathrm{bar}}^{P_{\mathrm{g}_{z+1}}} \alpha_{\mathrm{m}} V_{\mathrm{m}} \mathrm{d} P-\int_{1 \mathrm{bar}}^{P_{z}} \alpha_{\mathrm{m}} V_{\mathrm{m}} \mathrm{d} P_{\mathrm{g}}\right. \\
& \left.-\frac{1}{T_{z}}\left(\int_{1 \text { bar }}^{P_{z}} V_{\mathrm{m}} \mathrm{d} P-\int_{1 \text { bar }}^{P_{\mathrm{g}_{z+1}}} V_{\mathrm{m}} \mathrm{d} P_{\mathrm{g}}\right)\right]_{\left(P_{z}\right)} \\
& +\left.\sum_{\mathrm{m}} S_{\mathrm{m}_{z+1}}\left(n_{\mathrm{m}_{z}}-n_{\mathrm{m}_{z+1}}\right)\right|_{\left(T_{z+1}, P_{z+1}\right)},
\end{aligned}
$$

where the integration over the moles of the components is done at $P_{z+1}, T_{z+1}$, the integration over temperature from $T_{z+1}$ to $T_{z}$ at $n_{z}, P_{z+1}$ and the integration over pressure from $P_{z+1}$ to $P_{z}$ at $n_{z}, T_{z}$. The integral over pressure is better evaluated as the difference between the integral from 1 bar to $P$ at $z$ and from 1 bar to $P$ at $z+1$. The above equation can be rearranged using the relation

$$
S_{\mathrm{m}_{z+1}}=S_{\mathrm{m}_{z+1}}^{*}-\frac{1}{T_{z+1}}\left(\int_{z+2}^{z+1} V_{\mathrm{m}} \mathrm{d} P-\int_{z+2}^{z+1} V_{\mathrm{m}} \mathrm{d} P_{\mathrm{g}}\right)_{(z+1)}
$$


and the final expression for $S^{*}$ at $z$ after some substitutions is

$$
\begin{aligned}
S^{*}\left(P_{z}, T_{z}\right)= & \sum_{\mathrm{m}} n_{\mathrm{m}_{z}} S_{\mathrm{m}_{z+1}}^{*} \\
& +\sum_{\mathrm{m}} n_{\mathrm{m}_{z}}\{\cdots\} \\
& +\sum_{\mathrm{m}}\left(S_{\mathrm{m}_{z}}-S_{\mathrm{m}_{z+1}}\right)\left(n_{\mathrm{m}_{z}}-n_{\mathrm{m}_{z+1}}\right),
\end{aligned}
$$

where the quantity within the brackets $\{\ldots\}$ is the same as in Eq. (A10). The integrals with the heat capacity at 1 bar in Eqs. A3 and A4 and the integration of the volume at $T$ (Eqs. A3, A4, A10-A12) are solved analytically. The integration of $\alpha V$ over pressure is solved numerically. At a given temperature the numerical integration using the trapezoidal rule (Press et al., 1997) is

$$
\begin{aligned}
& \left.\int_{1 \mathrm{bar}}^{P} \alpha V \mathrm{~d} P\right|_{T} \approx \\
& \left.\left(\sum_{P_{i}} \alpha_{1 \mathrm{bar}} \frac{\left(V_{P_{i-1}}+V_{P_{i}}\right)}{2}+\frac{\left(V_{P_{i-1}} \Delta \alpha_{P_{i-1}}+V_{P_{i}} \Delta \alpha_{P_{i}}\right)}{2}\right)\right|_{T} \Delta P,
\end{aligned}
$$

where the index for the molar properties has been dropped for simplicity. The summation is over an arbitrary pressure grid ( $\Delta P=20 \mathrm{kbar}$ ) from 1 bar to the final pressure $P$. The quantity defined as $\Delta \alpha$ is the pressure contribution to the thermal expansion which is computed numerically at a certain pressure using the following reciprocity relation (Denbigh, 1971) in discretized form $\left(\Delta T=10^{\circ} \mathrm{C}\right)$ :

$$
\left.\frac{\partial \alpha}{\partial P}\right|_{T, P_{i}} \approx\left(\frac{1}{K_{T, P_{i}}^{2}}\right) \frac{\left(K_{T+\Delta T, P_{i}}-K_{T-\Delta T, P_{i}}\right)}{2 \Delta T}
$$

and then

$\Delta \alpha_{i} \approx \Delta \alpha_{i-1}+\frac{\left(\left.\frac{\partial \alpha}{\partial P}\right|_{T, P_{i}}+\left.\frac{\partial \alpha}{\partial P}\right|_{T, P_{i+1}}\right)}{2} \Delta P$,

where $\Delta \alpha_{i}$ is the total change of thermal expansion due to the pressure effect from 1 bar to $P_{i}$.

To evaluate $S^{*}$, the heat capacity in Eq. (A10) and similarly in the expressions for $H^{*}, S$ and $H$ should be computed at the pressure and temperature associated with the depth point $z+1$. However, the thermodynamic database (Saxena, 1996) is not suitable for precise evaluation of the pressure dependence of $C_{\mathrm{p}}$ (via integration of $\mathrm{d} C_{\mathrm{p}} /\left.\mathrm{d} P\right|_{T}=$ $-T \mathrm{~d}^{2} V /\left.\mathrm{d} T^{2}\right|_{P}$, Lewis and Randall, 1961). This is a common problem of existing databases (Jacobs et al., 2006; Tirone, 2015). The simplest but crude approximation is to take the heat capacity at 1 bar and at the given temperature. An alternative is that one can assume a linear pressure dependence between the heat capacity at 1 bar and the DulongPetit limit at some predefined high pressure value. This is based on the observation that at high pressure and temperature, $C_{\mathrm{p}}$ and $C_{\mathrm{v}}$ approach the Dulong-Petit value (Tirone, 2015). Both cases have been considered for the evaluation of a mantle geotherm in Sect. 5 .

The temperature that maintains the functions $S^{*}$ and $H^{*}$ at a constant level is found using a simple bisection method (Press et al., 1997). The whole algorithm is applicable for a reversible or irreversible adiabatic volume change. As mentioned in Sect. 2 when the scaling factor $\zeta$ is set to 1.0, $S^{*}$ (Eq. A3) reduces to the standard definition of entropy (Eq. A1) and $H^{*}$ is such that $\mathrm{d} H^{*}=C_{\mathrm{p}} \mathrm{d} T-\alpha V T \mathrm{~d} P=0$. One could imagine evaluating the thermodynamic properties at any depth following the same integration scheme from $1 \mathrm{bar}, 298 \mathrm{~K}$, that was applied for the bottom depth point (Eqs. A1-A5) instead of applying Eq. (A12). However for comparison with the reference bottom point value, it would have been necessary to carry on the slightly more difficult integration of the molar change term (for example for $\mathrm{S}$ or $S^{*}$, the expression $\sum \int S_{\mathrm{m}} \mathrm{d} n_{\mathrm{m}}$ at the final $P, T$ ).

Acknowledgements. This work benefited from discussions developed over several years with J. Ganguly. Clarity of some of the concepts presented in this study was greatly improved thanks to the feedback from the students of a graduate course in thermodynamics and geodynamics that has been offered at the Institut für Geologie, Mineralogie und Geophysik, Ruhr-Universität Bochum (2013-2015). Constructive comments of an anonymous reviewer and support of the topical editor (J. Afonso) are greatly appreciated.

Edited by: J. C. Afonso

\section{References}

Andrault, D., Muñoz, M., Bolfan-Casanova, N., Guignot, N., Perrillat, J.-P., Aquilanti, G., and Pascarelli, S.: Experimental evidence for perovskite and post-perovskite coexistence throughout the whole D region, Earth Plan. Sc. Lett., 293, 90-96, 2010.

Bird, R. B., Stewart, W. E., and Lightfoot, E. N.: Transport Phenomena, 2nd Edn., John Wiley and Sons, New York, USA, 895 pp., 2002.

Callen, H. B.: Thermodynamics and an Introduction to Thermostatics, 2nd Edn., John Wiley and Sons, New York, USA, 493 pp., 1985.

Denbigh, K.: The Principles of Chemical Equilibrium: With Applications in Chemistry and Chemical Engineering, 3rd Edn., Cambridge University Press, Cambridge, UK, 494 pp., 1971.

Dodson, M. H.: Isenthalpic flow, Joule-Kelvin coefficients and mantle convection, Nature, 234, p. 212, 1971.

Dorfman, S. M., Meng, Y., Prakapenka, V. B., and Duffy., T. S.: Effects of Fe-enrichment on the equation of state and stability of $(\mathrm{Mg}, \mathrm{Fe}) \mathrm{SiO}_{3}$ perovskite, Earth Plan. Sc. Lett., 361, 249-257, 2013.

Ganguly, J.: Adiabatic decompression and melting of mantle rocks: an irreversible thermodynamic analysis, Geophys. Res. Lett., 32, GL022363, doi:10.1029/2005GL022363, 2005. 
Ganguly, J.: Thermodynamics: Principles and Applications to Earth and Planetary Sciences, 1st edn., Springer, Berlin, Germany, New York, USA, 719 pp., 2008.

Hirose., K.: Postperovskite phase transition and its geophysical implications, Rev. Geophys., 44, RG3001, doi:10.1029/2005RG000186, 2006.

Jacobs, M. H. G., van den Berg, A., and de Jong, H. W. S: The derivation of thermo-physical properties and phase equilibria of silicate materials from lattice vibrations: Application to convection in the Earth's mantle, Calphad, 30, 131-146, 2006.

Komabayashi, T., Hirose, K., Sugimura, E., Sata, N., Ohishi, Y., and Dubrovinsky, L. S.: Simultaneous volume measurements of postperovskite and perovskite in $\mathrm{MgSiO}_{3}$ and their thermal equations of state, Earth Plan. Sc. Lett., 265, 515-524, 2008.

Lewis, G. N. and Randall, M.: Thermodynamics, 2nd Edn., McGraw Hill, New York, USA, 723 pp., 1961.

Manthilake, G. M., de Koker, N., Frost, D. J., and McCammon, A.: Lattice thermal conductivity of lower mantle minerals and heat flux from Earth's core, P. Natl. Acad. Sci. USA, 108, 1790117904, 2011.

McKenzie, D. and Bickle, M. J.: The volume and composition of melt generated by extension of the lithosphere, J. Petrol., 29, 625-679, 1988.

Murakami, M., Hirose, K., Kawamura, K., Sata., N., and Ohishi, Y.: Post-Perovskite phase transition in $\mathrm{MgSiO}_{3}$, Science, 304, 855858, 2004.

Press, W. H., Teukolsky, S. A., Vetterling, W. T., and Flannery, B. P.: Numerical Recipes in Fortran 77: The Art of Scientific Computing, 2nd Edn. (reprinted), Cambridge University Press, Cambridge, UK, 1486 pp. 1997.

Ramberg, H.: Temperature changes associated with adiabatic decompression in geological processes, Nature, 234, 539-540, 1971.

Sandler, S. I.: Chemical and Engineering Thermodynamics, 2nd edn., Wiley, New York, USA, 622 pp., 1988.

Saxena, S. K.: Earth mineralogical model: Gibbs free energy minimization computation in the system $\mathrm{MgO}-\mathrm{FeO}-\mathrm{SiO}_{2}$, Geochim. Cosmochim. Acta, 60, 2379-2395, 1996.
Schubert, G., Turcotte, D. L., and Olson, P.: Mantle Convection in the Earth and Planets, 1st Edn., Cambridge University Press, Cambrige, UK, 940 pp., 2001.

Shieh, S. R., Duffy, T. S., Kubo, A., Shen, G., Prakapenka, V. B., Sata, N., Hirose, K., and Ohishi, Y.: Equation of state of the postperovskite phase synthesized from a natural $(\mathrm{Mg}, \mathrm{Fe}) \mathrm{SiO}_{3}$ orthopyroxene, Proc. Natl. Acad. Sci. 103, 3039-3043, 2006.

Shim, S.-H.: The Postperovskite Transition, Annu. Rev. Earth Planet. Sci. 36, 569-599, 2008.

Spera, F. J.: Carbon dioxide in igneous petrogenesis: II. Fluid dynamics of mantle metasomatism, Contrib. Mineral. Petr., 77, 5665, 1981.

Spera, F. J., Yuen, D. A., and Giles, G.: Tradeoffs in chemical and thermal variations in the post-perovskite phase transition: Mixed phase regions in the deep lower mantle?, Phys. Earth Planet Int., 159, 234-246, 2006.

Tateno, S., Hirose, K., Sata., N., and Ohishi, Y.: Solubility of FeO in $(\mathrm{Mg}, \mathrm{Fe}) \mathrm{SiO}_{3}$ perovskite and the post-perovskite phase transition, Phys. Earth Planet Int., 160, 319-325, 2007.

Tirone, M.: On the use of thermal equations of state and the extrapolation at high temperature and pressure for geophysical and petrological applications, Geophys. J. Int., 202, 1483-1494, 2015.

Tirone, M., Ganguly, J., and Morgan, J. P.: Modeling petrological geodynamics in the Earth's mantle, Geochem. Geophy. Geosy., 10, 1-28, 2009.

Tsuchiya, J., Tsuchiya, T., and Wentzcovitch, M.: Vibrational and thermodynamic properties of $\mathrm{MgSiO}_{3}$ postperovskite, J. Geophys. Res., 110, B02204, doi:10.1029/2004JB003409, 2005.

Turcotte, D. L. and Schubert, G.: Geodynamics Applications of Continuum Physics to Geological Problems, 1st edn., Wiley and Sons, New York, USA, 450 pp., 1982.

Waldbaum, D. R.: Temperature changes associated with adiabatic decompression in geological processes, Nature, 232, 545-547, 1971.

Zemansky, M. W., Abbott, M. M., and Van Ness, H. C.: Basic Engineering Thermodynamics, 2nd Edn., McGraw-Hill, USA, 491 pp., 1975. 